\title{
POSSIBILITY OF FLUORESCENCE PHENOMENA IN THE ULTRAVIOLET SPECTRUM OF SYMBIOTIC STARS AND LONG PERIOD VARIABLES
}

\author{
J. P. SWINGS and P.SWINGS \\ Institut d'Astrophysique, Cointe-Ougrée, Belgium
}

\begin{abstract}
We discuss essentially the cases where molecular fluorescences may be excited by Lyman $\alpha$ and other strong discrete ultraviolet emissions, including lines beyond the Lyman limit. The stars involved are the symbiotic objects and the long period variables. The molecules are $\mathrm{H}_{2}, \mathrm{~N}_{2}, \mathrm{O}_{2}, \mathrm{NO}$ and $\mathrm{CO}$ which have their resonance systems in the ultraviolet.
\end{abstract}

\section{Introduction}

Atomic fluorescence phenomena are known to play a very important role in different celestial objects: in particular selected lines of OIII, NIII and HeII are present in nebulae and in high excitation bright-line stars. The excitation of these lines is due to resonance lines of HI and HeII. Such observations are of importance since they give information on certain emission lines which, lying beyond $912 \AA$, will never be directly detectable because of the interstellar absorption in the Lyman continuum (except in the case of quasars). Atomic fluorescence occurs also in other elements of hot stars, such as CIII, NIV, SiIII, SiII and possibly Fe II* giving rise to 'selectivities' among the lines of these elements.

Long period variables also show abnormal relative intensities due to the fluorescent excitation by lines located in the region $<3000 \AA$, hence not observable from the ground. This is the case for FeI and MnI excited by MgII $2795 \AA$, and for others excited by Mg 2851 and Sir (2516-2519 $\AA$ ).

We have examined the possibility of an excitation of molecular lines in certain types of stars by the discrete emissions of these stars. One could thus obtain information on molecules such as $\mathrm{H}_{2}, \mathrm{~N}_{2}, \mathrm{CO}, \ldots$ for which the resonance series are located in the far ultraviolet. The exciting lines may lie shortward of $912 \AA$.

\section{Emission of Molecular Lines in the UV Spectrum of Symbiotic Stars and Long Period Variables}

Certain stars which are rich in molecules also possess strong emission lines. This is the case of long period variables near minimum; they show emission lines of hydrogen, iron, ... in the observable spectrum although the temperature is low, of the order of $2000 \mathrm{~K}$. The intensity of their continuous spectrum should be very weak in the UV.

* UV FeII: Fluorescence may possibly explain the quartet-sextet selectivity. Observation of P Cygni stars will thus be of particular interest, especially in the region 2000-3000 $\AA$.

Houziaux and Butler (eds.), Ultraviolet Stellar Spectra and Ground-Based Observations, 226-231.

All Rights Reserved. Copyright (C) 1970 by the IAU. 
This would apply also to other cool stars, such as T Tauri stars. Symbiotic objects constitute another class of objects which combine emission lines and molecular absorption bands.

The characteristic difference between the spectra of long period variables and symbiotic objects concerns the excitation energy of the emission lines: this excitation is low in LPV's and may be very high in symbiotic stars.

The following considerations may be applied qualitatively to both groups. We shall examine them separately later.

In both groups the Lyman lines may be intense in emission since the Balmer series appears in emission. Evidently the Lyman series is not necessarily brighter than the Balmer series. On the other hand regions of the stellar atmospheres showing strong $\mathrm{TiO}$ or other molecular bands are certainly also rich in diatomic molecules (or sometimes triatomic, like $\mathrm{H}_{2} \mathrm{O}$ ) of cosmically abundant elements: $\mathrm{H}_{2}, \mathrm{~N}_{2}, \mathrm{O}_{2}, \mathrm{NO}, \mathrm{CO}$, .... All these molecules have their absorption electronic systems in the ultraviolet.

The absorption* of an emission line by a molecule having the appropriate values of the vibrational $\left(v^{\prime \prime}\right)$ and rotational $\left(K^{\prime \prime}\right)$ quantum numbers will give rise, through a fluorescence mechanism, to the emission of what is called a resonance series which will be composed of triplets, doublets or singlets depending on the type of the involved transition (see Swings, 1933).

If an emission line covers several absorption lines (this may be the case for a wide emission) the resonance series consists of multiplets resulting from the addition of triplets, doublets and singlets that characterize a unique absorption.

\section{A. WHAT ARE THE MOLECULAR FLUORESCENCES WHICH WE MAY EXPECT?}

A striking case results from the absorption of Lyman $\gamma$ by the nitrogen molecule $\mathrm{N}_{2}$. In order to observe Lyman $\gamma$ in the solar UV spectrum one must launch rockets higher than approximately $140 \mathrm{~km}$ : otherwise Lyman $\gamma$ is obscured by a telluric line of $\mathrm{N}_{2}$. At the same altitude, solar Lyman $\alpha$ and Lyman $\beta$ are already intense.

Let us examine a few possible cases of molecular fluorescence.

\section{1. $\mathrm{H}_{2}$ molecule}

The Lyman system of $\mathrm{H}_{2}$ may be excited by 8 emission lines as shown in Table $\mathrm{I}$; the Werner system could give rise to resonance series of triplets excited by lines also given in Table I. The transition probabilities are known in these cases and one may thus predict the intensity distribution in these resonance series.

\section{2. $\mathrm{N}_{2}$ molecule}

The absorption of Lyman $\gamma$ by $\mathrm{N}_{2}$ gives rise to a resonance series of triplets. The electronic transition of the Birge-Hopfield system is $b^{1} \Pi_{\mathrm{u}}-X^{1} \Sigma_{\mathrm{g}}^{+}$; the components of the triplets would be $\mathrm{P}(6), Q(5)$ and $R(4)$ of the series $\left(3, v^{\prime \prime}=0,1,2, \ldots\right)$ (Table II).

* The relative radial velocities of the molecular zones and of the exciting line zones must be taken into account. The problem is similar to that existing in comets (Swings, 1965). 
However, the intensity variation as a function of $v^{\prime \prime}$ is slow so that the energy available will be distributed through many triplets that individually will be weak.

Other resonance series of $\mathrm{N}_{2}$ may be excited by the discrete emissions given in Table II.

TABLE I

Possible fluorescences of $\mathrm{H}_{2}$

(a) Lyman system $B^{1} \Sigma_{\mathrm{u}^{+}-X^{1}} \Sigma_{\mathrm{g}}{ }^{+}$

\begin{tabular}{lll}
\hline$\lambda 1085.1$ & HeII & $P(6)-R(4)$ of the transitions $\left(2, v^{\prime \prime}\right)$ \\
$\lambda 1085.7$ & NII & $P(3)-R(1)$ O \\
$\lambda 1037.61$ & OvI & $P(1)$ of $\left(5, v^{\prime \prime}\right)$ or \\
& & $P(4)-R(2)$ of $\left(6, v^{\prime \prime}\right)$ \\
$\lambda 1031.9$ & OvI & $P(6)-R(4)$ of $\left(6, v^{\prime \prime}\right)$ \\
$\lambda 1025.7$ & Ly $\beta$ & $P(1), P(3)-R(1)$ and \\
& & $P(4)-R(2)$ of $\left(6, v^{\prime \prime}\right)$ \\
$\lambda 977.03$ & CIII & $P(5)-R(3)$ of $\left(11, v^{\prime \prime}\right)$ \\
$\lambda 972.54$ & Ly $\gamma$ & $P(3)-R(1)$ of $\left(11, v^{\prime \prime}\right)$ \\
$\lambda 949.74$ & Ly $\delta$ & $P(2)-R(0)$ of $\left(14, v^{\prime \prime}\right)$ and \\
& & $P(7)-R(5)$ of $\left(15, v^{\prime \prime}\right)$
\end{tabular}

(b) Werner system $C^{1} \Pi_{\mathrm{u}}-X^{1} \Sigma_{\mathrm{g}}+$

\begin{tabular}{|c|c|c|c|}
\hline$\lambda$ & 989.8 & NiII & $P(4)-Q(3)-R(2)$ of $\left(1, v^{\prime \prime}\right)$ \\
\hline$\lambda$ & 977.03 & CIII & $\begin{array}{l}P(5)-Q(4)-R(3) \\
P(7)-Q(6)-R(5)\end{array}$ of $\left(2, v^{\prime \prime}\right)$ \\
\hline$\lambda$ & 949.74 & Ly $\delta$ & $\begin{array}{l}P(6)-Q(5)-R(4) \\
P(2)-Q(1)-R(0)\end{array}$ of $\left(3, v^{\prime \prime}\right)$ \\
\hline$\lambda$ & 937.80 & Ly $\varepsilon$ & $P(4)-Q(3)-R(2)$ of $\left(4, v^{\prime \prime}\right)$ \\
\hline$\lambda$ & 930.75 & Ly $\zeta$ & $P(2)-Q(1)-\mathrm{R}(0)$ of $\left(4, v^{\prime \prime}\right)$ \\
\hline
\end{tabular}

TABLE II

Possible fluorescences of $\mathrm{N}_{2}$

(a) Resonance series excited by Lyman $\gamma$ $b^{1} \Pi_{\mathrm{u}}-X^{1} \Sigma_{\mathrm{g}}{ }^{+}$system (Birge-Hopfield)

\begin{tabular}{lrrr}
\hline Transition & \multicolumn{1}{l}{$P(6)$} & $Q(5)$ & \multicolumn{1}{l}{$R(4)$} \\
\hline$(3,0)$ & 972.54 & 972.32 & 972.13 \\
$(3,1)$ & 995.09 & 994.85 & 994.65 \\
$(3,2)$ & 1018.41 & 1018.16 & 1017.96 \\
$(3,3)$ & 1042.55 & 1042.28 & 1042.07 \\
$(3,4)$ & 1067.53 & 1067.25 & 1067.03 \\
$(3,5)$ & 1093.39 & 1093.11 & 1092.87
\end{tabular}

(b) Resonance series excited by:

\begin{tabular}{llll}
\hline$\lambda 949.74$ & Ly $\delta$ & $P(7)-Q(6)-R(5)$ of $\left(6, v^{\prime \prime}\right)$ in $b^{1} \Pi_{\mathrm{u}}-X^{1} \Sigma_{\mathrm{g}}{ }^{+}$ \\
$\lambda 937.80$ & Ly $\varepsilon$ & $P(2)-R(0)$ & of $\left(4, v^{\prime \prime}\right)$ in $b^{1} \Sigma_{\mathrm{u}}{ }^{+}-X^{1} \Sigma_{\mathrm{g}}{ }^{+}$ \\
\hline
\end{tabular}


One may not exclude an excitation of $\mathrm{N}_{2}$ by the Lyman continuum; in such a case the fluorescent emissions would correspond to numerous rotation transitions and would look like laboratory emission bands.

3. $\mathrm{O}_{2}$ molecule

$\mathrm{O}_{2}$ will probably not be observable because of the predissociation effect in the Schumann-Runge system and the weakness of the transition probabilities.

\section{NO molecule}

The only possibility of a discrete excitation seems to be that by NIII (1098.11 $\AA$ ) which falls in the $\delta$ system $c^{2} \Sigma-\mathrm{X}^{2} \Pi(0,0)$ (band head at $1910 \AA$, degraded toward the violet).

\section{5. $\mathrm{CO}$ molecule}

(a) Fourth positive system $A^{1} \Pi-X^{1} \Sigma$. A strong CIV line (1546 $\AA$ ) is situated near the head of the $(0,0)$ band. The corresponding rotational quantum number is $J=32$. One may expect an emission of triplets $\left(0, v^{\prime \prime}\right)$, especially $(0,3)$ and $(0,4)$.

Other possibilities of coincidences may be found on the basis of a new vibrational and rotational study of the fourth positive system of CO in the UV (Simmons et al., 1969): for example Ni $(1200 \AA)$ lies near $1199.67 \AA(15,0)$ and CIII $1175 \AA$ near $1173.15 \AA(17,0)$.

(b) Fluorescence could also possibly be excited in the Birge-Hopfield systems: the only two possible coincidences seem to be:

N II $(1087.7 \AA)$ (strong line) which falls in the band $(0,0)$ of $c^{1} \Sigma^{+}-X^{1} \Sigma^{+}$; and Lyman $\delta(949.7 \AA)$ which falls in $G^{1} \Pi-X^{1} \Sigma^{+}$.

However the rotational analysis of the $\mathrm{CO}$ bands is still too incomplete to enable a discussion of the fluorescence possibilities.

\section{6. $\mathrm{H}_{2} \mathrm{O}$ molecule}

A possible excitation of polyatomic molecules may occur only in very cold stars, i.e. in the coldest part of symbiotic objects or of long period variables.

The far ultraviolet spectrum of $\mathrm{H}_{2} \mathrm{O}$ has not yet been sufficiently analysed to enable a prediction of fluorescence phenomena. Furthermore the absorption spectrum of $\mathrm{H}_{2} \mathrm{O}$ shows bands that seem diffuse or continuous: fluorescence excited in such regions would thus be weak or continuous. It should be noted that certain bands may simply have a continuous appearance, yet have a discrete structure.

The Lyman $\alpha$ line is absorbed by $\mathrm{H}_{2} \mathrm{O}$ but we do not know whether or not this absorption is followed by a fluorescent emission of $\mathrm{H}_{2} \mathrm{O}$. It is possible that the absorption by $\mathrm{H}_{2} \mathrm{O}$ leads to a dissociation or predissociation that would produce an emission of atomic lines or $\mathrm{OH}$ bands.

\section{B. WHICH ARE THE LINES PRODUCING AN EXCITATION?}

In the case of long period variables, only Lyman lines and, possibly, a few lines of rather low excitation such as OI or Ni may be considered. On the contrary, in sym- 
biotic objects, we may also consider an excitation by permitted resonance and recombination lines and forbidden lines of high excitation, such as HeI, HeII, OII, OIII, [OIII].... The possible exciting lines are summarized in Table III (from Moore, 1952). A list of UV lines of planetary nebulae has been published by Aller (1961).

TABLE III

Strongest exciting UV lines (resonance and recombination)

\begin{tabular}{lrrl}
\hline CI & CII & \multicolumn{1}{c}{ CIII } & CIV \\
\hline 1657 & 1335 & 977 & $1548-50$ \\
1561 & 1037 & 386 & 312 \\
1277 & 904 & 1175 & 420 \\
1193 & 858 & 538 & 384 \\
& 687 & 459 & \\
& & 2297 & \\
\hline
\end{tabular}

\begin{tabular}{|c|c|c|c|c|c|c|}
\hline $\mathrm{NI}_{\mathrm{I}}$ & NII & NIII & Niv & Nv & & \\
\hline 1200 & 1084 & 990 & 765 & \multicolumn{2}{|c|}{$1238-42$} & \\
\hline 1134 & 916 & 764 & 247 & \multicolumn{2}{|c|}{209} & \\
\hline 964 & 672 & 686 & 923 & & \\
\hline 1493-95 & 645 & 452 & 322 & & & \\
\hline $1743-45$ & 776 & 374 & 283 & & & \\
\hline 1412 & & 772 & 1719 & & & \\
\hline $1327-28$ & & & 955 & & & \\
\hline 1311 & & & 335 & & & \\
\hline OI & OII & OIII & & Orv & $\mathrm{Ov}$ & OVI \\
\hline $1355-58$ & $833-34$ & $833-3$ & & $788-90$ & 630 & $1032-38$ \\
\hline $1302-06$ & 539 & $702-0$ & & 609 & 172 & 150 \\
\hline $1039-42$ & 718 & $507-0$ & & 554 & $759-62$ & 184 \\
\hline $1026-28$ & 538 & 374 & & 280 & 192 & 173 \\
\hline 990 & & 306 & & 239 & 220 & \\
\hline & & 603 & & 625 & & \\
\hline & & 600 & & & & \\
\hline & & 526 & & & & \\
\hline & & 396 & & & & \\
\hline & & 328 & & & & \\
\hline & & 321 & & & & \\
\hline & & 598 & & & & \\
\hline & & 435 & & & & \\
\hline & & 345 & & & & \\
\hline
\end{tabular}

\section{Conclusion}

Long period variables, despite their low temperature, may reveal an ultraviolet spectrum rich in emission lines of molecular origin. Since the ultraviolet continuum of the LPV is most probably very weak, only excitation by discrete emission lines may be 
considered in these stars. Symbiotic objects may show a more complex UV spectrum because of the presence of a greater number of discrete exciting lines. An ultraviolet continuum, combined with discrete absorption and emission lines may also be present in symbiotic objects and give rise to more complex fluorescence emissions.

It is impossible to determine quantitatively the intensities of the molecular fluorescence lines: this is due to the lack of information on geometrical and physical models for the stars considered here and also on the relative radial velocities. One thing is however certain: the intensities of the molecular lines will be much weaker than those of the exciting lines. Even if the energy of an exciting line is entirely absorbed by a molecule the resonance series which is produced is composed of several lines, generally numerous lines. For instance the intensity of each line of the resonance series of $\mathrm{N}_{2}$ excited by Lyman $\gamma$ is only a small fraction of the intensity of Lyman $\gamma$.

In any case observations from rockets or satellites will be of great interest.

\section{References}

Aller, L. H.: 1961, Mem. Soc. Roy. Sc. Liège 4, 535.

Moore, C. E.: 1952, A UV Multiplet Table, NBS Circ. 488.

Simmons, J. D., Bass, A. M., and Tilford, S. G.: 1969, Astrophys. J. 155, 345.

Swings, P.: 1933, 'Spectres moléculaires', Act. Sc. Ind. 74, Paris, Hermann.

Swings, P.: 1965, 'Astronautical Investigations of Comets', in Advances in Space Science and Technology, Vol. 7 (ed. by F. I. Ordway III), Academic Press, New York.

\section{Discussion}

Underhill: If the cool gas where the molecular bands originate was irradiated by an absorption-line UV spectrum from a hot object (perhaps in the case of symbiotic stars), would not the bands show dips of intensity at some places, the lines of relevant energy not being excited.

Swings: It seems that long period variables may be represented by a stratified model. Emissions of hydrogen, [FeII] and $\mathrm{AlO}$ take place in different regions. This can be seen by measuring the relative intensities of the lines and comparing them to theoretical values. In symbiotic objects and in long period variables, it happens often that $\mathrm{H} \beta$ in emission is very weak because of its absorption by TiO. This means that hydrogen lines are emitted in lower layers. In this configuration it is easily noticed that atomic emission lines may excite molecules lying above them. A paper discusses this stratification problem of the emitting layers in long period variables (J. P. Swings: 1969, Bull. Soc. Roy. Sc. Liège, in press).

Malaise: I would like to stress a point that was mentioned in the paper. When discussing comparisons between observed emission spectra and model atmosphere results in the UV, it should be kept in mind that if molecules are present, they can absorb selectively one emission line and redistribute the absorbed photons over such a number of lines that they simply appear to be washed out. This process could change the observed intensity ratios of emission lines in the UV. 